\title{
Relação entre a estimativa de dose de radiação em pacientes submetidos a exames de tomografia computadorizada do abdômen e o índice de massa corporal
}

\author{
A. S. Capaverde a; J. Pimentel a; A. P. P. Froner ${ }^{\text {a; }}$ A. M. M. da Silva ${ }^{\text {b }}$ \\ ${ }^{a}$ PUCRS, Hospital São Lucas, 90610-000, Porto alegre-RS, Brasil \\ ${ }^{b}$ PUCRS, Faculdade de Física, 90619-900, Porto alegre-RS, Brasil
}

alexandre.capaverde@acad.pucrs.br

\section{RESUMO}

Devido à dose de radiação na tomografia computadorizada (TC) ser relativamente alta, é importante que se tenha uma estimativa da dose a que o paciente é submetido, considerando parâmetros utilizados e fatores de correção, a fim de que o valor seja mais próximo do real. O objetivo deste estudo é relacionar a estimativa de dose em pacientes submetidos à TC do abdômen com os grupos IMC, considerando o tamanho específico da região anatômica. O trabalho desenvolveu-se em um hospital de Porto Alegre, utilizando equipamento Siemens Somaton Emotion 16. Foram coletados 30 indivíduos adultos que realizaram TC do abdômen em Janeiro de 2014. Destes, 17 realizaram aquisição utilizando mecanismo de redução de dose (Care Dose) (Amostra 1) e o restante sem este mecanismo (Amostra 2). Registrou-se peso, altura, $C T D I_{\text {vol }}$ e diâmetro lateral e ântero-posterior na altura do umbigo. Foram calculados valores de IMC e o fator de correção para a estimativa de dose, de acordo com o tamanho específico do abdômen. Determinou-se a variação percentual entre os valores de $C T D I_{v o l}$ fornecidos pela TC e o valor do $C T D I_{v o l}$, após aplicação do fator de correção, além da variação percentual média para cada grupo IMC. A variação percentual média ficou entre 65\% e 19\% para a Amostra 1 e entre 35\% e 10\% para a Amostra 2, do menor para o maior valor do grupo IMC. Observou-se redução da variação percentual média com o aumento do grupo IMC, em ambas as amostras. É necessária uma amostra maior de indivíduos para verificação dos resultados encontrados.

Palavras-chave: Tomografia Computadorizada; Dose de radiação; IMC. 


\begin{abstract}
Because of the radiation dose in computed tomography (CT) is relatively high, it is important to have an estimate of the dose to which the patient is submitted, considering parameters and correction factors, with objective of value is closer to the real. The objective of this study is to relate the dose estimation in patients undergoing abdominal CT with BMI groups, considering the specific size of the anatomical region. The work was developed in a hospital in Porto Alegre, using Siemens Somatom Emotion 16. We collected 30 adults who underwent abdominal CT in January 2014. Of these, 17 underwent acquisition using dose reduction mechanism (Care Dose) (Sample 1), and the

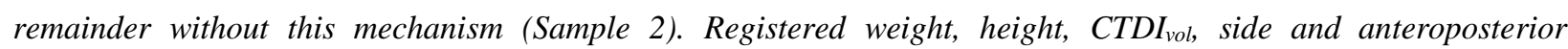
diameter at the navel. We were calculated BMI values and the correction factor for dose estimation, according to the specific size of the abdomen. It was determined the percentage change between the CTDI vol values provided by

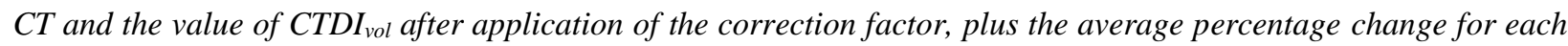
BMI group. The average percentage variation was between 65\% and 19\% for Sample 1 and between 35\% and 10\% for Sample 2, the lowest to highest BMI group. There was a reduction in the average percent change with increasing BMI groups in both samples. A larger sample of individuals is required for verification of results.
\end{abstract}

Keywords: Computed Tomography; Radiation Dose; BMI.

\title{
1. INTRODUÇÃO
}

A tomografia computadorizada (TC) é uma técnica de diagnóstico por imagem que utiliza radiação ionizante para aquisição de cortes transversais ao longo de um determinado volume. A instalação destes equipamentos no Brasil vem crescendo de forma significativa nos últimos anos [1] e, consequentemente, o número de exames realizados também cresceu. Em vista disso, é importante que se tenha um controle sobre a quantidade de radiação a que os pacientes são submetidos. Na TC existe um conjunto de grandezas para quantificar a exposição à radiação, entre eles estão o índice de dose na tomografia computadorizada (CTDI, do inglês Computed Tomography Dose Index) e o produto dose comprimento (DLP, do inglês Dose Length Product) [2]. Para cada exame realizado, a TC informa o valor do CTDI volumétrico $\left(C T D I_{v o l}\right)$ que expressa uma estimativa da dose de radiação, em mGy, recebida em um determinado volume. 
Entretanto, é importante levar em consideração que cada indivíduo submetido ao exame de TC possui características físicas próprias, como, por exemplo, peso e altura, o que resulta em uma diferença no seu índice de massa corporal (IMC). O IMC é definido como a razão entre o peso, em kg, e o quadrado da altura, em m, do indivíduo [3]. De acordo com a Organização Mundial da Saúde, o IMC pode ser dividido em grupos da seguinte forma: Baixo Peso, que apresenta um valor de IMC menor que $18,5 \mathrm{~kg} / \mathrm{m}^{2}$; Peso Normal, com IMC entre 18,5 e 24,9 kg/m²; Sobrepeso, com IMC maior ou igual a $25 \mathrm{~kg} / \mathrm{m}^{2}$; Pré-Obeso, com IMC entre 25,0 e 29,9 kg/m²; Obeso I, com IMC entre 30,0 e 34,9 kg/m²; Obeso II, que possui IMC entre 35,0 e 39,9 kg/m²; e Obeso III, com IMC maior ou igual a $40,0 \mathrm{~kg} / \mathrm{m}^{2}$ [3].

Segundo a Associação Americana de Física em Medicina (AAPM, do inglês American Association of Physicists in Medicine), a dose de radiação recebida por um indivíduo em um exame de TC é dependente do seu tamanho e da quantidade de radiação produzida pelo equipamento [4]. Porém, o $C T D I_{v o l}$ informado pelo equipamento fornece informações relativas unicamente à quantidade de radiação produzida [4]. Ou seja, não leva em consideração o peso, a altura ou o IMC de cada indivíduo. O $C T D I_{v o l}$ fornecido pelo equipamento é definido para um simulador de referência, isto é, um simulador cilíndrico de crânio, que possui diâmetro de 16,0 cm, ou de corpo, com diâmetro de 32,0 cm, o qual é selecionado pelo equipamento dependendo do protocolo de aquisição utilizado [4]. A partir disso, a AAPM desenvolveu fatores de conversão que podem ser aplicados ao $C T D I_{v o l}$, permitindo que se obtenha uma estimativa mais próxima do valor real de dose recebido pelo indivíduo [4]. Estes fatores de correção se baseiam no tamanho específico da região anatômica a ser estudada como, por exemplo, o diâmetro equivalente do crânio, tórax ou abdômen.

McCollought e colaboradores [5] destaca que o $C T D I_{v o l}$ e a dose que o paciente recebe não representam a mesma medida. O $C T D I_{v o l}$ informa precisamente a operação do equipamento. Este valor deve ser utilizado em conjunto com as informações sobre o tamanho e anatomia do paciente, para estimar a dose. 
Christner e colaboradores [6], Brady e Kaufman [7] utilizaram da publicação da AAPM para a conversão do $C T D I_{v o l}$, levando em consideração o tamanho específico da região anatômica em pacientes adultos que realizaram TC de tórax e em pacientes pediátricos, respectivamente. No entanto, nenhum destes pesquisadores separou suas amostras por características antropomórficas.

Rodrigues e colaboradores [8] relacionaram os valores de dose recebida em pacientes submetidos a exames de TC do abdômen com as suas características antropomórficas, entre elas o IMC. Seu estudo concluiu que há um aumento da dose efetiva à medida que o valor do IMC dos pacientes aumenta [8]. Porém, não foi utilizado nenhum fator de conversão dos dados coletados para o tamanho específico da região anatômica estudada.

Este trabalho teve como objetivo relacionar a estimativa de dose de radiação em indivíduos submetidos à TC do abdômen com os respectivos grupos IMC, considerando o tamanho específico desta região anatômica. Para tanto, foi coletada uma série de dados relativos a um grupo de indivíduos que realizou exames TC do abdômen, sendo os valores de dose recebida convertidos utilizando a recomendação da $A A P M$ [4], e por fim, relacionar estes dados os respectivos grupos IMC. Este trabalho foi analisado e aprovado pelo Comitê de Ética em Pesquisa (CAAE 18924113.0.0000.5336).

\section{MATERIAIS E MÉTODOS}

Este trabalho foi desenvolvido em um grande hospital de Porto Alegre, utilizando um equipamento de TC fabricado pela empresa Siemens, modelo Somaton Emotion 16. A metodologia foi divida da seguinte forma: (1) avaliação da acurácia dos descritores de dose; (2) coleta de dados relacionados à amostra; (3) avaliação e análise dos dados coletados. 


\subsection{Acurácia dos descritores de dose}

Para avaliação da acurácia dos descritores de dose no equipamento de TC, foi utilizado um simulador de abdômen fabricado de polimetilmetacrilato (PMMA), com 32,0 cm de diâmetro, comercializado pela empresa Nuclear Associates. Além do simulador, foi utilizada uma câmara de ionização tipo lápis com 100,0 mm de comprimento e um eletrômetro, ambos fabricados pela empresa Radcal. Foram realizadas 3 medidas em 5 pontos diferentes do simulador, sendo estes: centro (C), superior (S), inferior (I), direita (D) e esquerda (E). A Figura 1 mostra uma representação do simulador e dos diferentes pontos para a inserção da câmara de ionização.

Figura 1: Representação do simulador e dos diferentes pontos de inserção da câmara de ionização.
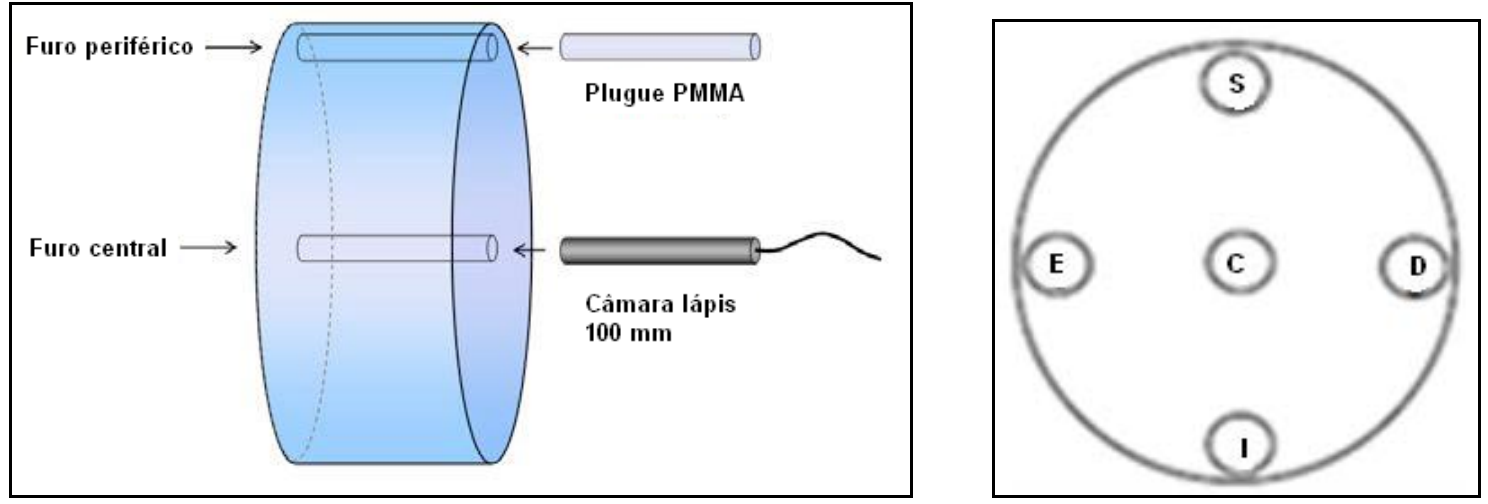

Fonte: AAPM Report 204. [4]

Para o cálculo do $C T D I_{v o l}$ através das medidas realizadas, é necessário realizar o cálculo do CTDI ao longo do comprimento da câmara de ionização. Este parâmetro é denominado $C T D I_{100 \text {, }}$ e pode ser obtido através da equação 1 [2].

$$
C T D I_{100}=\left(\frac{1}{N T}\right) \int_{-50}^{50} D_{\text {único }}(z) d z
$$


Onde $\mathrm{N}$ é o número de secções adquiridas ao longo da varredura, $\mathrm{T}$ é a espessura nominal de cada secção e Dúnico é o valor de dose registrado no eletrômetro. Para cada um dos 5 pontos medidos no simulador, é calculado o valor do $C T D I_{100}$. Um segundo índice de dose foi criado que fornece uma média ponderada das contribuições do centro e da periferia dentro do plano de varredura [2]. Este índice é chamado $C T D I_{w}$ e calculado conforme a equação 2 [2].

$$
C T D I_{w}=\left(\frac{1}{3}\right)\left(C T D I_{100}\right)_{\text {centro }}+\left(\frac{2}{3}\right)\left(C T D I_{100}\right)_{\text {periférico }}
$$

Para o cálculo do $C T D I_{v o l}$ deve-se levar em consideração o pitch (fator de passo) que pode ser descrito como razão entre a distância percorrida pela mesa durante uma rotação $360^{\circ}$ e a espessura total colimada do feixe de raios X [2]. O CTDI $I_{v o l}$ é definido pela equação e tem como unidade o mGy [2].

$$
C T D I_{v o l}=\frac{C T D I_{w}}{\text { pitch }}
$$

Outro parâmetro utilizado para avaliação da dose de radiação na TC é o $D L P$, que é definido como a dose recebida pelo volume ao longo do comprimento de varredura (d), em centímetros. A equação 4 é utilizada para o cálculo do DLP e tem como unidade o mGy.cm [2].

$$
D L P=C T D I_{v o l} \times d
$$




\subsection{Coleta de dados}

Através do sistema de informação e armazenamento de imagens do hospital foram coletados 30 indivíduos que realizaram TC do abdômen durante o mês de Janeiro de 2014. Deste grupo, 17 indivíduos realizaram a aquisição das imagens utilizando um mecanismo de redução de dose (Care Dose 4D), sente estes denominados Amostra 1, e 13 sem este mecanismo, sendo denominados Amostra 2. De acordo com Rodrigues e colaboradores [8], o CARE Dose 4D "permite modular a corrente do tubo de acordo com as dimensões do paciente”. Ou seja, dependendo das dimensões do paciente, este mecanismo ajusta os valores de corrente elétrica (mA) ao longo da varredura reduzindo a dose de radiação total recebida.

Para cada indivíduo selecionado, foram coletadas as seguintes informações pessoais e do protocolo de aquisição: peso $(\mathrm{kg})$, altura $(\mathrm{m})$, tensão $(\mathrm{kV})$, corrente elétrica $(\mathrm{mA})$, pitch, $C T D I_{v o l}$ (mGy) e DLP (mGy.cm). A partir destes valores foram calculados os valores de IMC e da estimativa da dose efetiva recebida. Uma estimativa da dose efetiva (E) na região anatômica que passou pela varredura pode ser obtida através da multiplicação do $D L P$ por um fator de correção que leva em consideração a região anatômica irradiada. Para o abdômen, este fator de correção $\mathrm{f}$ é igual a 0,015 mSv/mGy.cm [9]. Assim, para o cálculo da dose efetiva na região do abdômen utiliza-se a equação 5 .

$$
E=0,015 \times D L P
$$

A tabela 1 mostra os valores de referência, para a região do abdômen, dos descritores de dose utilizados na TC, de acordo com o European Guidelines on Quality Criteria for Computed Tomography [9]. 
Tabela 1: Valores de referência para o $C T D I_{v o l}, D L P$ e Dose Efetiva.

\begin{tabular}{cccc} 
& $\boldsymbol{C T D I}_{\text {vol }}(\mathbf{m G y})$ & $\boldsymbol{D L P}(\mathbf{m G y} . \mathbf{c m})$ & Dose Efetiva (mSv) \\
\hline Abdômen & 35 & 780 & 11,7
\end{tabular}

Fonte: European guidelines on quality criteria for computed tomography [9]

Seguindo a recomendação da $A A P M$ na sua publicação número 204 [4] de 2011, foi medido, em cada paciente, o diâmetro lateral (LAT) e ântero-posterior (AP) na altura do umbigo. A figura 2 ilustra como esta medida deve ser realizada nas imagens de TC bem como a tabela com os fatores de conversão.

Figura 2: Medidas de diâmetro lateral e ântero-posterior nas imagens de TC do abdômen (esq.) e tabela com fatores de conversão (dir.).
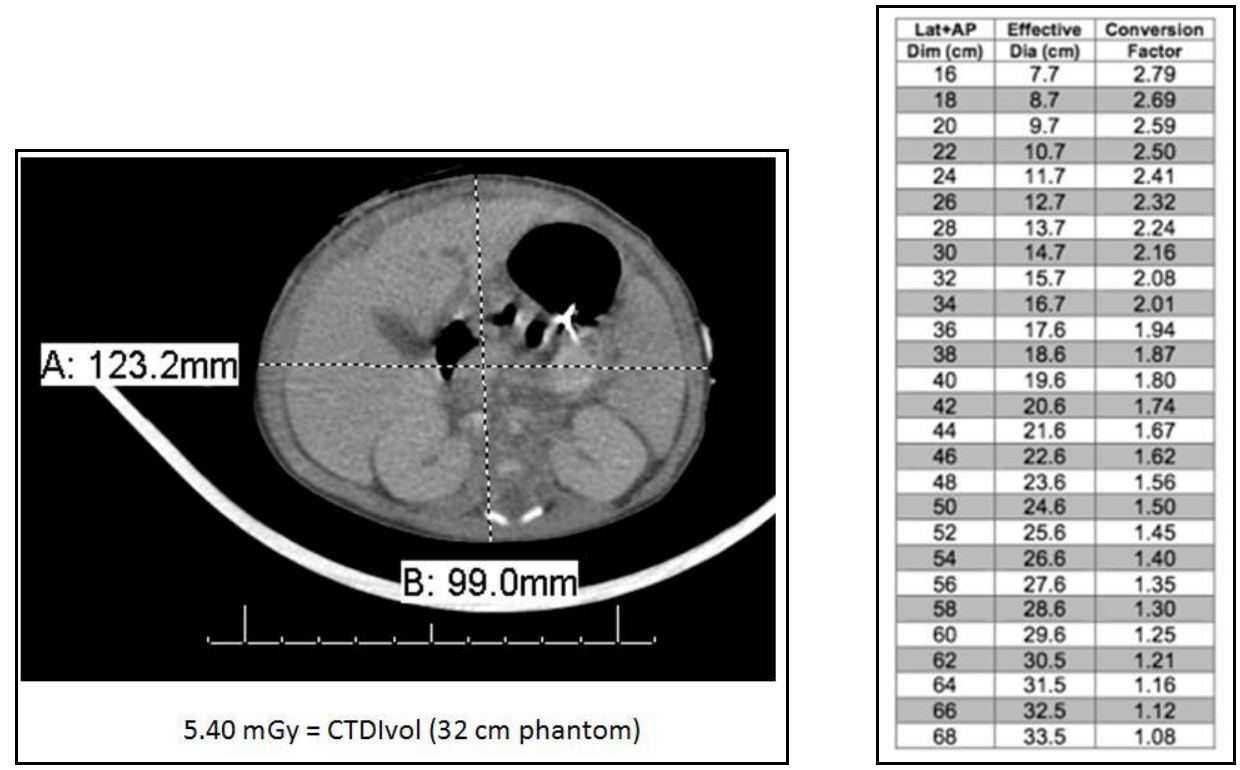

Fonte: AAPM Report 204 [4]

Para determinar o fator de conversão, é necessário somar os dois diâmetros medidos (LAT + AP) e verificar na tabela fornecida pela AAPM o valor indicado para a região anatômica determinada. Na figura 2 à esquerda, são fornecidos os valores dos dois diâmetros (123,2 mm e 99,0 mm) e ainda o valor do $C T D I_{\text {vol }}(5,4 \mathrm{mGy})$ de acordo com um simulador de $32,0 \mathrm{~cm}$. À direta é 
apresenta a tabela com os valores da soma dos diâmetros, em cm, e o fator de conversão. No caso da figura 2, o resultado da soma dos diâmetros é igual a 22,0 cm e verificando na tabela ao lado encontra-se um fator de conversão de 2,50. Este fator de conversão deve ser multiplicado pelo $C T D I_{v o l}$ para determinar a estimativa de dose para o tamanho específico (do inglês, SizeSpecific Dose Estimates (SSDE)) da região anatômica. No caso da figura acima, o valor do SSDE é igual a 13,5 mGy [4].

Os procedimentos para medir os diâmetros LAT e AP, além da determinação do SSDE, foram realizados para cada indivíduo coletado. As medidas dos diâmetros foram feitas utilizando o software de visualização de imagens fornecido pelo hospital.

\subsection{Avaliação e análise dos dados coletados}

Após a coleta dos dados, e da determinação dos valores de SSDE para cada indivíduo, as duas amostras foram organizadas de acordo com os respectivos valores de IMC e separadas em grupos. Foi calculado para cada indivíduo a variação percentual entre os valor de $C T D I_{v o l}$ fornecido pelo equipamento e o valor do $C T D I_{v o l}$ após a aplicação do fator de conversão (SSDE). Por fim, determinou-se a variação percentual média para cada um dos grupos IMC. Para a avaliação e análise dos dados foi utilizada uma planilha de cálculos.

\section{RESULTADOS}

Os valores encontrados para as medidas realizadas na avaliação da acurácia dos descritores de dose da TC encontram-se na tabela 2. Observou-se uma variação entre o valor indicado no equipamento e o valor medido de $11,09 \%$. 
Tabela 2: Valores obtidos para o teste de acurácia dos descritores de dose.

\begin{tabular}{cc}
\cline { 2 - 2 } & $\boldsymbol{C T D I}_{\text {vol }}(\mathrm{mGy})$ \\
\hline $\begin{array}{c}\text { CTDI } \\
\text { (indicado) } \\
\text { CTDI } \\
\text { (medido) } \\
\text { Diferença } \\
\text { percentual (\%) }\end{array}$ & 14,35 \\
\hline
\end{tabular}

Com relação aos dados coletados dos indivíduos e a relação com os respectivos valores de IMC, para a amostra 1, obteve-se 3 indivíduos no grupo IMC Baixo Peso; 6 para o grupo Peso Normal; 4 para o grupo Pré-Obeso e 4 para o grupo Obeso I. Em relação à amostra 2, verificou-se 6 indivíduos para o grupo Peso Normal; 6 para o grupo Pré-Obeso e 1 para o grupo Obeso II. A tabela 3 (A) mostra os valores de $C T D I_{\text {vol }}$ e $S S D E$ encontrados para os indivíduos da amostra 1, bem como os valores da variação percentual média para cada grupo IMC. Na tabela 3 (B) encontram-se os valores obtidos para os parâmetros no amostra 2.

Tabela 3: Valores obtidos de $C T D I_{v o l}, S S D E$ e da variação percentual média em relação aos grupos IMC para: (A) Amostra 1; (B) Amostra 2.

\begin{tabular}{ccccc}
\hline Grupo IMC & $\begin{array}{c}\text { Razão média } \\
\text { entre o diâmetro } \\
\text { LAT e AP (cm) }\end{array}$ & $\begin{array}{c}\mathbf{C T D I} \\
\mathbf{( m G o l ~ m e ́ d i o ~}\end{array}$ & $\begin{array}{c}\text { SSDE médio } \\
(\mathbf{m G y})\end{array}$ & $\begin{array}{c}\text { Variação } \\
\text { percentual } \\
\text { média (\%) }\end{array}$ \\
\hline Baixo Peso & $1,44 \pm 0,17$ & $6,49 \pm 1,17$ & $9,86 \pm 1,24$ & $54 \pm 8$ \\
Peso Normal & $1,50 \pm 0,14$ & $8,34 \pm 1,10$ & $12,87 \pm 1,29$ & $55 \pm 7$ \\
Pré-Obeso & $1,34 \pm 0,03$ & $9,27 \pm 0,75$ & $12,20 \pm 0,84$ & $32 \pm 5$ \\
Obeso I & $1,43 \pm 0,01$ & $13,86 \pm 2,73$ & $16,25 \pm 2,33$ & $19 \pm 6$ \\
\hline
\end{tabular}




\begin{tabular}{ccccc}
\hline Grupo IMC & $\begin{array}{c}\text { Razão média } \\
\text { entre o diâmetro } \\
\text { LAT e AP (cm) }\end{array}$ & $\begin{array}{c}\text { CTDI } \begin{array}{c}\text { ( Bol médio } \\
(\mathbf{m G y})\end{array} \\
\text { Peso Normal }\end{array}$ & $\begin{array}{c}\text { SSDE médio } \\
\mathbf{( m G y )}\end{array}$ & $\begin{array}{c}\text { Variação } \\
\text { percentual } \\
\text { média (\%) }\end{array}$ \\
\hline Pré-Obeso & $1,34 \pm 0,23$ & $14,53 \pm 0$ & $19,64 \pm 1,09$ & $35 \pm 7$ \\
Obeso I & $1,45 \pm 0,10$ & $14,53 \pm 0$ & $18,67 \pm 1,10$ & $29 \pm 7$ \\
Obeso II & $1,23 \pm 0,00$ & $14,53 \pm 0$ & $16,42 \pm 0,15$ & $13 \pm 1$ \\
\hline
\end{tabular}

A figura 3 mostra um gráfico da dose efetiva média em função dos grupos IMC para as amostras 1 e 2. Em vermelho é possível observar os valores obtidos para a amostra 2, nos respectivos grupos IMC, e em azul os valores obtidos para a amostra 1, também nos respectivos grupos IMC.

Figura 3: Gráfico da dose efetiva média em função dos grupos IMC.

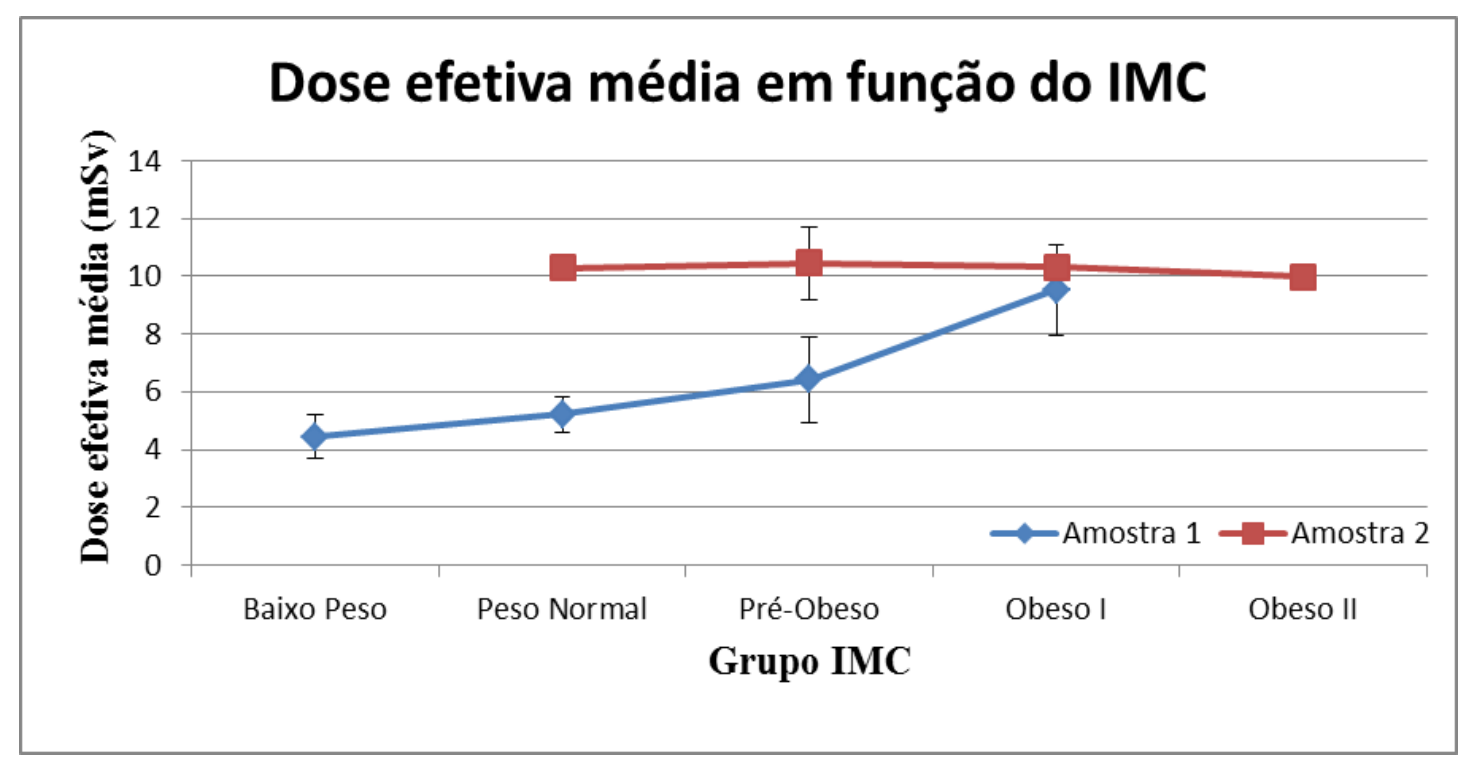

Por fim, é apresentado um gráfico da variação percentual média entre os valores de CTDI $I_{v o l}$ informados pelo equipamento e do SSDE obtido em relação aos grupos IMC. No gráfico, é possível verificar em azul os valores para a Amostra 1 e, em vermelho, os valores para a Amostra 2. 
Figura 4: Gráfico da variação percentual média entre o $C T D I_{v o l}$ e o $S S D E$ em função dos grupos IMC.

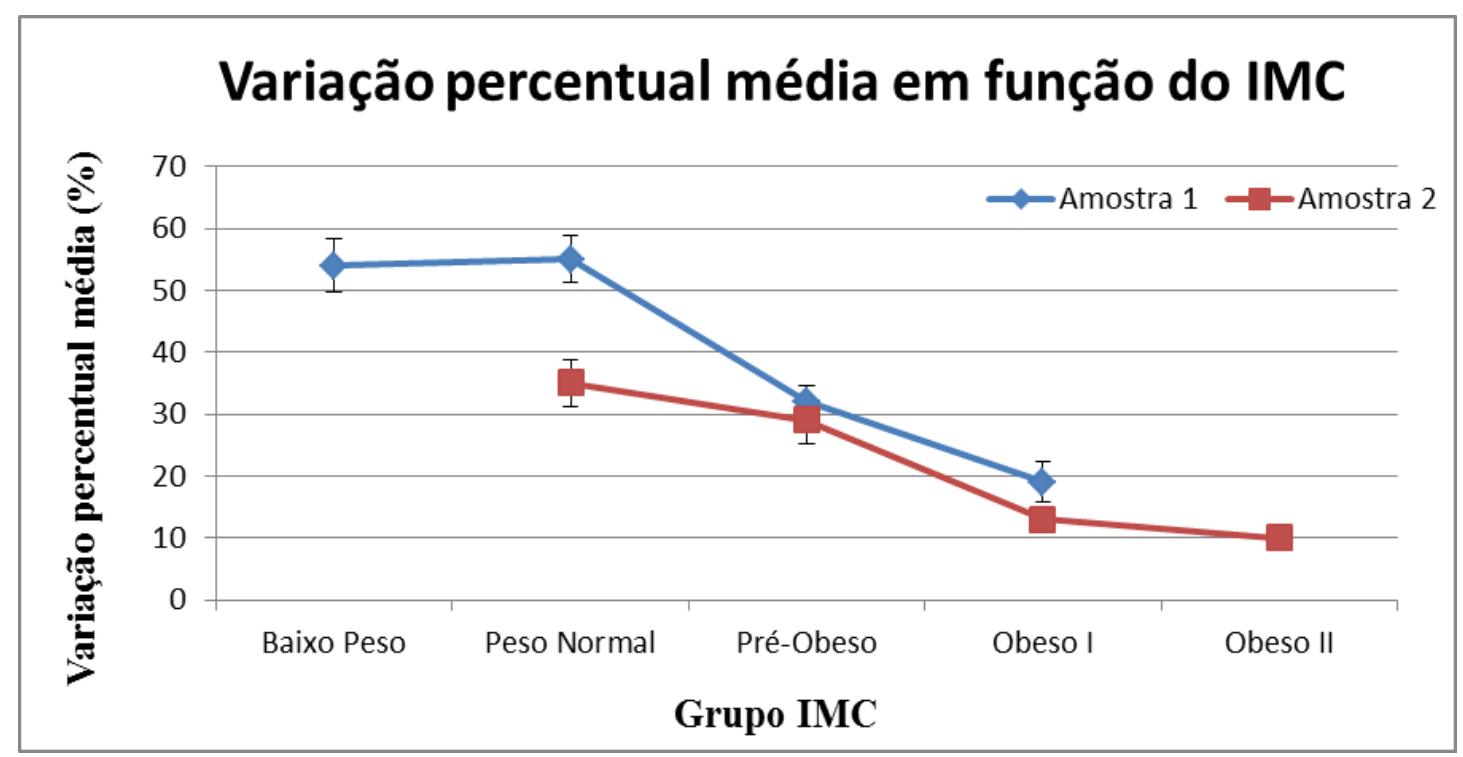

\section{DISCUSSÃO}

Verificou-se que os resultados obtidos para a avaliação da acurácia dos descritores de dose em equipamentos de TC encontravam-se dentro dos limites estabelecidos pelo European Guidelines on Quality Criteria for Computed Tomography [9], conforme a tabela 1. A diferença percentual entre o $C T D I_{\text {vol }}$ medido e o valor mostrado no equipamento foi de $11 \%$, indicando que pode haver uma certa discrepância entre a dose produzida e a informada no comando do equipamento, ou ainda possíveis erros de medida associados ao uso do eletrômetro ou da câmara de ionização durante a aquisição dos dados. É necessária uma quantidade maior de testes e mais precisos a fim de verificar a constância dos valores encontrados. Entretanto, cabe ressaltar que os valores encontrados estão abaixo dos níveis de limite de dose de radiação para pacientes, conforme publicações internacionais e nacionais. 
Em relação aos dados coletados, foi possível concluir que para a Amostra 1, que utiliza o mecanismo CARE Dose $4 D$, os valores de $C T D I_{v o l}$ médio foram menores do que os valores obtidos para a Amostra 2, que não utilizou o mecanismo de redução. Devido ao fato que este mecanismo altera os valores de corrente elétrica ao longo da varredura [8], o resultado encontrase dentro do esperado. Para a Amostra 2, os valores de $C T D I_{v o l}$ médio se mantiveram praticamente constantes, independente do diâmetro do indivíduo ou do seu grupo IMC. Isso faz com que a dose recebida por um indivíduo que tenha um valor de IMC menor seja aproximadamente a mesma de um individuo com IMC maior, causando uma superexposição, além do desgaste maior do equipamento. O valor de dose que este indivíduo recebeu foi obtido levando-se em consideração somente a dose produzida pelo equipamento, que foi definida com um simulador cilíndrico com 32,0 cm de diâmetro. O peso, a altura e o valor do IMC foram desconsiderados. Aplicando-se o fator de conversão recomendado pela $A A P M$ [4], é possível perceber que a dose de radiação recebida pelos indivíduos da Amostra 2 foi maior que aquela recebida pelos indivíduos da Amostra 1, devido ao fato da não ser utilizado o mecanismo de redução de dose. A dose efetiva média recebida pelos indivíduos da Amostra 1 cresceu conforme o grupo IMC aumentou, corroborando os dados obtidos por Rodrigues e colaboradores [8]. Porém, para a Amostra 2, a dose efetiva média se manteve praticamente constante com o aumento do IMC, mostrando que este valor praticamente não se altera de indivíduo para indivíduo.

A relação entre a variação percentual média e os grupos IMC ocorreu de forma decrescente com o aumento dos valores de IMC dos indivíduos. Para baixos grupos IMC, a variação percentual média foi maior que as verificadas para valores altos de IMC. Na Amostra 1, a variação percentual média para o grupo IMC Peso Normal foi cerca de 54\% a mais do que o valor informado pelo equipamento TC. Ainda na Amostra 1, a mesma variação para o grupo IMC Obeso I foi cerca de 19\%. Esta diferença é justificada através da razão entre o diâmetro lateral e o diâmetro ântero-posterior destes indivíduos. Em indivíduos que pertencem aos menores grupos IMC, a razão entre os seus diâmetros é superior a 1, devido ao fato destes valores não serem iguais, ou seja, a anatomia de cada individuo influencia este valor. Nestes indivíduos, o diâmetro 
lateral é superior ao diâmetro ântero-posterior. À medida que os valores de IMC aumentam, a razão entre os diâmetros destes indivíduos se aproxima de 1, conforme verificado na tabela 3, devido ao raio da circunferência abdominal possuir praticamente o mesmo valor ao longo da anatomia. Devido ao fato do $C T D I_{v o l}$ ser calibrado utilizando um simulador de dose cilíndrico, no qual a razão entre os seus diâmetros é igual a 1, quanto mais próximo de 1 é a razão entre os diâmetros do indivíduo, mais próxima a dose informada pelo equipamento é daquela à qual o indivíduo realmente é submetido. Este fato foi verificado para as duas amostras, sendo que a variação percentual média foi maior para a Amostra 1 do que para a Amostra 2.

Por fim, a relação entre a estimativa de dose de radiação em pacientes submetidos a exames de TC do abdômen e o IMC, levando em consideração o tamanho específico desta região anatômica, mostrou-se uma alternativa para a avaliação da real dose a que estes indivíduos são submetidos. Utilizando os fatores de conversão recomendados pela $A A P M$ para conversão do valor do $C T D I_{v o l}$ informado pelo equipamento [4], concluiu-se que esta ferramenta é de extrema importância em indivíduos com baixos valores de IMC, onde a variação percentual média entre o valor informado e o corrigido foi cerca de $54 \%$.

\section{CONCLUSÃO}

Faz-se necessário que uma amostra maior de indivíduos seja analisada para a verificação dos resultados encontrados neste trabalho, porém, com base nas evidências encontradas, pode-se afirmar que o IMC é um fator a ser levado em consideração nos exames de TC do abdômen, principalmente para pacientes pertencentes aos grupos de mais baixo valor. Novas investigações estão sendo realizadas em relação a outros aspectos relacionados a fatores antropomórficos que podem influenciar na dose de radiação, como descrito por Rodrigues e colaboradores [8], como o perímetro abdominal e a massa corporal. 


\section{AGRADECIMENTO}

Agradecemos ao Centro de Diagnóstico por Imagem do Hospital São Lucas da PUCRS por disponibilizar os dados e os equipamentos para realização deste trabalho.

\section{REFERÊNCIAS}

1. IBGE - Instituto Brasileiro de Geografia e Estatística. Indicadores Sociodemográficos e de Saúde no Brasil 2009: Escassez e fartura: distribuição da oferta de equipamentos de diagnóstico por imagem no Brasil. Brasil, v. 1, 26 p. Disponível em:

$<$ http://www.ibge.gov.br/home/estatistica/populacao/indic_sociosaude/2009/com_esca.pdf > Último acesso: 20 de janeiro de 2015.

2. MCNITT-GRAY, M. F. AAPM/RSNA Physics Tutorial for Residents: Topics in CT. Radiation Dose in CT. RadioGraphics, v. 22, p. 1541-1553, 2002. Disponível em: < http://www.ncbi.nlm.nih.gov/pubmed/12432127>. Último acesso: 20 de janeiro de 2015.

3. ABESO - Associação Brasileira para o estudo da obesidade e da síndrome metabólica. Diretrizes Brasileiras de Obesidade. $\quad 3^{\text {a }}$ ed. São Paulo: AC Farmacêutica, 2009. Disponível em:

< http://www.abeso.org.br/pdf/diretrizes_brasileiras_obesidade_2009_2010_1.pdf>. Último acesso: 20 de janeiro de 2015.

4. AAPM - American Association of Physicists in Medicine. Size-Specific Dose Estimates (SSDE) in Pediatric and Adult Body CT Examinations. AAPM Report 204, 2011. 30 p. 
Disponível em: < http://www.aapm.org/pubs/reports/rpt_204.pdf>. Último acesso: 20 de janeiro de 2015.

5. MCCOLLOUGH, C. H. , et al. CT Dose Index and Patient Dose: They are not the same thing. Radiology 259, v. 2, p. 311-316, 2011. Disponível em:

<http://pubs.rsna.org/doi/abs/10.1148/radiol.11101800>. Último acesso: 20 de janeiro de 2015.

6. CHRISTNER, J. A. , et al. Size-specific Dose Estimates for Adult Patients at CT of the Torso. Radiology 265, v. 3, p. 841-847, 2012. Disponível em:

< http://pubs.rsna.org/doi/abs/10.1148/radiol.12112365>. Último acesso: 20 de janeiro de 2015.

7. BRADY, S. L., KAUFMAN, R. A. Investigation of American Association of Physicists in Medicine Report 204 Size-specific Dose Estimates for Pediatric CT Implementation. Radiology 265, v. 3, p. 832-840, 2012. Disponível em:

$<$ http://pubs.rsna.org/doi/full/10.1148/radiol.12120131>. Último acesso: 20 de janeiro de 2015.

8. RODRIGUES, S. I. , et al. Estudo da Dose nos Exames de Tomografia Computadorizada Abdominal em um Equipamento de 6 Cortes. Radiologia Brasileira 45, v. 6, p. 326-333, 2012. Disponível em:

< http://dx.doi.org/10.1590/S0100-39842012000600008>. Último acesso: 20 de janeiro de 2015.

\section{EUR - European Comission. European Guidelines on Quality Criteria for Computed}

Tomography. EUR 16262 EN. 1998, 114 p. Disponível em:

$<$ http://w3.tue.nl/fileadmin/sbd/Documenten/Leergang/BSM/European_Guidelines_Quality_Crit eria_Computed_Tomography_Eur_16252.pdf>. Último acesso: 20 de janeiro de 2015. 